\title{
Les difficultés de l'OLAF pour s'imposer en tant qu'acteur légitime de la protection des intérêts économiques et financiers européens
}

\section{Véronique Pujas}

\section{(2) OpenEdition}

Journals

Édition électronique

URL : http://journals.openedition.org/conflits/2076

DOI : $10.4000 /$ conflits.2076

ISSN : $1777-5345$

Éditeur :

CCLS - Centre d'études sur les conflits lilberté et sécurité, L'Harmattan

Édition imprimée

Date de publication : 1 juin 2006

Pagination : 107-127

ISBN : 2-296-01073-3

ISSN : 1157-996X

Référence électronique

Véronique Pujas, «Les difficultés de l'OLAF pour s'imposer en tant qu'acteur légitime de la protection des intérêts économiques et financiers européens », Cultures \& Conflits [En ligne], 62 | printemps 2006, mis en ligne le 20 juillet 2006, consulté le 30 mars 2021. URL : http://journals.openedition.org/conflits/ 2076 ; DOI : https://doi.org/10.4000/conflits.2076

Ce document a été généré automatiquement le 30 mars 2021

Creative Commons License 


\title{
Les difficultés de l'OLAF pour
} s'imposer en tant qu'acteur légitime de la protection des intérêts économiques et financiers européens

\author{
Véronique Pujas
}

1 «L'OLAF est le fruit de circonstances exceptionnelles, elle est la conséquence du scandale et correspond à la nécessité de redonner de la crédibilité aux institutions européennes ${ }^{1}$ ".

2 Ce constat reste vrai aujourd'hui, alors qu'une réforme substantielle de l'OLAF 2, discutée dans le contexte de la nouvelle crise de 2004, semble désormais abandonnée.

3 En effet, c'est une crise politique aboutissant au refus du Parlement européen d'accorder la décharge budgétaire à la Commission qui marquera la première réforme majeure de la politique de lutte contre les fraudes de l'UE. La deuxième réforme envisagée fait suite à une deuxième vague de scandales, inaugurée par l'affaire Eurostat, et qui va se solder par une motion de censure. Celle-ci va, certes, échouer mais elle va remettre en cause une fois de plus l'efficacité de l'OLAF. Depuis, la Commission et les représentants nationaux ont ouvert une large consultation pour réformer l'office. Pourtant, à l'aube de 2006 et au regard de l'échec des ambitions de communautarisation de l'espace judiciaire européen, le projet de réforme s'est réduit comme une peau de chagrin, au risque de continuer à produire, dans les années à venir, les mêmes scénarios.

4 Au-delà de ses modalités d'évolution, l'OLAF est un objet institutionnel inédit, particulièrement intéressant à étudier, et ce pour plusieurs raisons, symptomatiques des nécessités politiques contemporaines. Il est au centre d'intérêts convergents concernant l'émergence et l'imposition d'un nouveau paradigme politique: la protection des intérêts financiers des communautés et la lutte contre les phénomènes 
de criminalité transnationaux qui y sont liés, mais aussi l'incarnation d'une ambition politique âprement discutée de la construction européenne, à savoir la création formelle d'un espace européen de justice et de sécurité intégré, dont le Procureur européen serait le contrôleur de légalité. Pourtant, dans cette tension entre communautarisation du paradigme sécuritaire européen et intergouvernementalité grâce à la coopération des contrôleurs nationaux, force est de constater que la seconde option a aujourd'hui le vent en poupe. La réforme avortée d'OLAF à ce stade consacre une tendance à la segmentation et à la parcellisation des initiatives, visant à créer une politique européenne de la sécurité peu cohérente et, surtout, vouée à des difficultés inextricables en termes de mise en ouvre des mesures décidées.

5 C'est ce que nous allons voir en expliquant le processus historico-institutionnel de la création de l'Unité de coordination anti-fraude (UCLAF) à la fin des années 1980 et sa mutation en office de lutte anti-fraude (OLAF), une décennie plus tard. Ses modalités institutionnelles sont encore discutées aujourd'hui, à la lumière de plusieurs affaires qui remettent en cause la pertinence de ses statuts fondateurs.

6 On s'intéressera, dans un premier temps, à l'émergence et à l'institutionnalisation de ce nouveau paradigme de l'action publique européenne - «la prévention et la répression des fraudes nuisant aux intérêts communautaires »- en s'interrogeant sur ses liens avec, d'une part, la création de l'UCLAF et, d'autre part, les évolutions récentes de son successeur, l'OLAF. Nous verrons dans un second temps que plusieurs polémiques, depuis sa mise en place, font douter de ses capacités à protéger les fonds européens mais, surtout, qu'il n'apparaît pas comme un acteur légitime dans le paysage européen. Enfin, le climat institutionnel et de crise politique européenne qui prévaut depuis 2002 laisse entrevoir de nouvelles orientations, non annoncées, de la politique de lutte contre les fraudes.

7 A partir de la mise sur agenda des problèmes de fraudes dans la Commission même, autant que la dénonciation de son inefficacité pour remédier à l'évasion des fonds communautaires au niveau des Etats membres, il s'agit d'identifier les acteurs du processus d'institutionnalisation de l'espace judiciaire européen, mais aussi de mettre l'accent sur leurs intérêts convergents et divergents. Deux hypothèses, respectivement néo-institutionnaliste et intergouvernementale, nous permettaient, en 2001, d'offrir un cadre général d'interprétation aux orientations prises en matière de lutte contre les fraudes au niveau européen ${ }^{3}$. Cinq années plus tard, nous privilégions la seconde, au vu de l'évolution de l'OLAF et de son cadre institutionnel dans le contexte actuel.

8 La recherche exposée dans cet article se situe à la croisée des politiques publiques et de la sociologie de l'action publique avec, en filigrane, des réflexions sur la sociologie des mobilisations. Elle s'appuie sur une série d'entretiens réalisée avec les personnels d'OLAF en 2000, lors de la première réforme, mais également sur le suivi systématique des polémiques qui se sont développées dans les médias (essentiellement dans les journaux en ligne comme europeanvoice.com, eupolitix.com et euobserver.com), ainsi qu'à travers la réflexion élaborée pour le rapport de la chambre des Lords qui a fait, en juillet 2004, une première évaluation de la nouvelle réforme proposée et discutée en $2005^{4}$.

9 D'un point de vue théorique, les hypothèses sous-jacentes sur lesquelles reposent les développements sont celles classiquement utilisées pour l'étude des affaires européennes, à savoir néo-institutionnaliste et intergouvernementale. Elles nous permettent de donner un cadre général d'interprétation aux orientations alternatives 
prises en matière de lutte contre les fraudes au niveau européen et, surtout, de tester laquelle des deux hypothèses prévaut selon les phases d'évolutions de l'OLAF. On verra ainsi que, bien que la phase d'institutionnalisation ait été stratégiquement conçue par la Commission et le Parlement européen pour supporter la vision communautaire d'un espace de sécurité et de justice européen, celle qui lui succède, à la faveur des échecs du traité de Nice et du traité de Convention européenne, opère un revirement vers la vision intergouvernementale et minimaliste de la coopération européenne sur les questions de criminalités financières.

L'émergence de l'UCLAF-OLAF et « la protection des intérêts économiques et financiers des communautés européennes"

10 Le processus historico-institutionnel présidant à l'émergence de l'Unité anti-fraude est lié à l'évolution institutionnelle de deux secteurs clé de la construction européenne : les compétences budgétaires et financières attribuées aux institutions supranationales européennes et le développement de l'intégration des Etats membres dans un espace judiciaire européen. Ces deux secteurs, relevant des 1er et 3e piliers du temple européen, ont suivi des développements très inégaux, qui se reflètent de toute évidence dans le « canard boiteux » que constitue cette politique européenne de lutte contre les fraudes.

11 L'imposition de l'idée selon laquelle les fraudes et corruptions observées au niveau communautaire dépendaient de la responsabilité des institutions européennes relève largement d'un artefact et non pas de l'objectivation des problèmes à résoudre. Pourtant, cette perception a dominé jusqu'en 2005. La construction de ce problème public est en réalité le résultat de tensions entre la stratégie de renforcement institutionnel en direction du supranational orchestrée par la Commission, le Parlement et le Conseil où s'exprime la politique des Etats membres. Ces derniers souhaitent renforcer l'intergouvernementalisme dans le cadre du 3e pilier, lequel est le véritable enjeu de la lutte contre les fraudes. D'une certaine manière, ils s'accommodent fort bien de l'idée selon laquelle l'institution supranationale européenne endossera la responsabilité d'une politique de la lutte contre les fraudes et corruptions, sujet politiquement périlleux dans les arènes nationales.

12 La prise de conscience par les acteurs des institutions supranationales communautaires que les fraudes et la corruption les concernent directement, se produit dans les années 1970, lorsque les institutions de la Communauté se voient dotées de "ressources propres ", indépendantes des Etats membres. Les problèmes de fraude, qui ont toujours existé, mais qui n'ont acquis une certaine visibilité que dans certaines conjonctures, sont alors présentés comme relevant logiquement des compétences communautaires et donc de la "community building", et non plus seulement des Etats membres. L'évolution institutionnelle et économique communautaire présidant à l'émergence et à la mise en forme de cette idée selon laquelle le niveau institutionnel supranational européen doit prendre en charge la question de la corruption et des fraudes communautaires, nuira profondément au traitement du problème, comme nous le verrons par la suite.

13 Dans la lignée de l'attribution de ressources budgétaires propres, est créée en 1978 la Cour des comptes européenne, une agence indépendante des Etats membres et des institutions communautaires, destinée à contrôler et à vérifier l'usage fait des dépenses communautaires. Dès le début des années 1980, les rapports de la Cour des comptes relèvent des irrégularités et des fraudes, notamment et surtout concernant le 
détournement des subventions agricoles (la politique agricole commune représente alors $70 \%$ des dépenses communautaires ${ }^{5}$ ). Ces rapports, communiqués et commentés devant les représentants élus par la Commission de contrôle budgétaire du Parlement européen (Cocobu), dénoncent les dysfonctionnements et fraudes dans leur gestion à plusieurs reprises, (successivement en $1984^{6}, 1994^{7}, 1995^{8}$ et en $1998{ }^{9}$, à l'occasion de la procédure de la décharge budgétaire). Compétente pour contrôler les comptes des institutions communautaires, la Cour des comptes n'est pas dotée de ressources d'investigation, ni de compétences suffisantes pour mener des enquêtes sur des problèmes de fraudes ${ }^{10}$. Consciente de cette lacune, elle est relayée par les rapports de la Cocobu, qui préconise une véritable action communautaire pour lutter contre l'évasion des fonds européens.

14 Sur la base d'un rapport interne relatif aux activités de lutte contre la fraude, la Commission décide, en 1987, de la création d'une Unité de coordination de la lutte antifraude (UCLAF) ${ }^{11}$, laquelle serait divisée en "cellules anti-fraude", distribuées entre les principaux services de dépenses et de recettes. Elle devient opérationnelle en juillet 1988. Les rapports successifs de la Cour des comptes font cependant mention d'irrégularités et de fraudes. Dans le cadre de la procédure de décharge de 1990 et de 1994 par le Parlement européen, ses compétences demeurent partagées entre les DG VI (agriculture, le budget le plus important de la CE), XIX (budget), XX (contrôle financier) et XXI (douanes et fiscalité directe), mais ses effectifs sont renforcés. En 1995, l'UCLAF est placée sous la responsabilité de la commissaire suédoise chargée des questions de contrôles budgétaires et financiers, Madame Gradin, qui devient également par conséquent responsable de la lutte contre la fraude. Lors de cette même année est décidée la centralisation des activités opérationnelles des services de l'UCLAF. Mais, en 1996, la commission d'enquête créée par le Parlement sur le régime de transit communautaire ${ }^{12}$, pilier de la libre circulation des marchandises et qui, à cause des fraudes, voit s'évader des millions d'euros, pointe déjà les défaillances et faiblesses de l'UCLAF. Elle recommande de développer le rôle de l'UCLAF « en tant que cellule de renseignement sur les délits pénaux » et de créer un «bureau central d'échanges d'information pour aider les Parquets nationaux dans leurs poursuites judiciaires à caractère transnational ».

15 A la faveur du traité de Maastricht, qui inscrit pour la première fois la lutte contre la criminalité organisée comme une ambition européenne, mais aussi menacés par la vague d'euroscepticisme qui commence à s'installer, les Etats membres, largement incités en cela par la Commission et le Parlement, reconnaissent dans le cadre des Conseils européens de Copenhague en 1993, leur manque de rigueur dans la gestion des fonds communautaires, et arrivent à un accord unanime sur le fait qu'ils doivent traiter les fraudes communautaires comme ils traitent les fraudes grevant leur propres budgets ${ }^{13}$. Une fois ces questions discutées à nouveau dans le cadre de la réunion des ministres de la Justice et des Affaires intérieures à Essen, en décembre 1994, les Etats membres sont exhortés à prendre des dispositions communes en la matière. Cette collaboration prend la forme d'une convention et d'un accord intergouvernemental, procédure nécessitant non seulement une ratification par les parlements des Etats membres pour entrer en vigueur, mais également une régulation du Conseil établissant un cadre juridique pour d'ultérieures actions communautaires dans ce domaine. Cette approche n'est pas soutenue par le Parlement européen qui préconisait, lui, une action renforcée sur la base du 1er pilier et, donc, sous le contrôle des institutions européennes. Un accord de principe est toutefois trouvé lors du Conseil européen de 
Corfou, en 1994, qui aboutit, en 1995, à l'adoption d'une Convention sur la protection des intérêts financiers des Communautés européennes ${ }^{14}$, définissant le cadre général des activités de la Commission dans la lutte contre la fraude ${ }^{15}$ Une politique européenne concertée de la lutte contre les fraudes est, de fait, prisonnière d'une double contingence. La lenteur des délais de ratification de la Convention et de ses protocoles additionnels signés en $1996{ }^{16}$ et $1997{ }^{17}$ (appelés « instruments PIF », relatifs à la protection des intérêts financiers des Communautés) donnant les moyens juridiques et légaux pour une telle action, rendent nulle la réalisation de la coopération européenne en cette matière. Ils font, par ailleurs, douter de la sincérité de la volonté politique des Etats membres de lutter effectivement contre les fraudes et corruptions. En effet, les dimensions pratiques des mesures de remboursement des fonds détournés et les poursuites judiciaires occasionnées par la découverte de ces irrégularités restent de la compétence des Etats membres, dont plusieurs, sur leur territoire, ne se distinguent pas par l'efficacité de leurs politiques nationales en matière de répression des problèmes de fraudes et corruptions ${ }^{18}$.

16 Le contexte est donc celui d'une prise de conscience politique de l'existence d'un problème (objectivé en tant que tel par les représentants des Etats membres au sein du Conseil), mais dont les mesures d'application demeurent essentiellement à la discrétion de ces mêmes Etats. La création de l'UCLAF, organisme communautaire chargé de la lutte contre la corruption au sein de la Commission,vient en appoint au contrôle financier interne existant déjà avec la DG XX. Cette innovation cristallise les tensions résultant des incohérences entre la construction du problème - une part des fonds communautaires sont détournés, il faut donc "protéger les intérêts financiers des communautés" - et les moyens adoptés pour le résoudre: la création d'une agence supranationale de détection des fraudes sans réelle indépendance ni moyens adéquats, dont les effets punitifs sont renvoyés à la responsabilité des Etats membres. Cette incohérence, placée dans un contexte institutionnel spécifique sur lequel nous reviendrons, débouchera sur la première crise du premier semestre 1999.

Malgré les diverses mutations de l'UCLAF entre 1987 et 1999, le renforcement de ses effectifs, la succession des déclarations de bonnes intentions relatives à la lutte contre les fraudes nuisant aux intérêts des communautés ${ }^{19}$, les services de contrôle financiers ne cessent de mettre en évidence la persistance de pratiques au contraire très "tolérantes", concernant les adaptations à la règle et ses détournements au sein des institutions européennes. "Les insuffisances relevées dans la mise au jour des irrégularités et des fraudes, ainsi que dans les enquêtes afférentes ", "l'absence de poursuites judiciaires efficaces et cohérentes à l'échelle de l'Union européenne » sont dénoncées par le rapport de la Cocobu et par son rapporteur, Herbert Bosch, le 7 octobre 1998. Le rapport conclut sur la nécessité de réformer l'UCLAF, vivement critiquée à l'intérieur même de la Commission. Celle-ci répondra par une première proposition, fidèle aux recommandations du rapport Bosch en décembre 1998. Toutefois, Jacques Santer fait part, le lendemain de sa démission le 15 mars 1999, d'une proposition modifiée en vue de la constitution de l'office de lutte anti-fraude. "Si en raison de la présence de l'UCLAF dans la structure des services de la Commission, nos efforts de lutte anti-fraude sont mis en cause ou dénigrés par d'autres institutions, alors il faut externaliser cette fonction d'enquête ${ }^{20}$ ». Interprétée comme une volonté de déconnecter l'Unité d'investigation des services contrôlés de ses sources d'informations - puisqu'il a seulement une vocation d'enquêtes administratives internes -, cette proposition irritera particulièrement les parlementaires européens, 
sensibles à une lutte effective contre les fraudes. Pourtant a posteriori, et au vu des derniers développements qui tendent à politiser l'office, cette solution parait la seule viable pour assurer l'indépendance de l'office.

Dans le cadre de l'exercice de la décharge budgétaire de 1998, les tensions provoquées par une conjonction de faits infirmant la volonté affichée de l'exécutif de lutter efficacement contre les pratiques de fraudes, aboutiront à la crise institutionnelle du premier semestre $1999^{21}$. Le Parlement européen exprimera sa défiance vis-à-vis de la Commission, en relevant précisément sa responsabilité dans le développement d'irrégularités et de pratiques de népotisme non réprimées, pointées par le rapport des auditeurs. Cela lui permet, par la même occasion, de consolider en pratique ses nouveaux pouvoirs en matière de co-décision et de contrôle budgétaire ${ }^{22}$, alors que son activité législative a décru ces dernières années, mais aussi d'attirer l'attention médiatique sur son activisme en matière de transparence dans le contexte électoral du printemps 1999. Une fois la décharge concernant le budget de 1998 repoussée, deux motions de censure seront votées. Mais aucune n'obtient une majorité suffisante pour être adoptée. La première donnera toutefois lieu à la constitution d'un comité d'experts indépendants, appelé « comité de sages », dont le rapport " apolitique et extérieur aux institutions communautaires » est destiné à " calmer le jeu » et à faire toute la lumière sur les accusations du Comité de contrôle budgétaire du Parlement. Les conclusions se révèleront des plus négatives et confirmeront les défaillances de la Commission pour prévenir et punir les déviances au sein de ses propres services. Ses conclusions déboucheront sur la démission du collège de commissaires placé sous la direction de Jacques Santer ${ }^{23}$. Le rapport Bosch sera adopté par le Parlement en mai 1999, confirmant la création de l'OLAF et l'adoption de la législation afférente selon la procédure de co-décision.

19 Comme nous l'avons vu, durant cette première phase d'institutionnalisation, la formulation du problème et l'imputation des responsabilités sont largement le fait des organes financiers européens (Cour des comptes, Cocobu, DG contrôle financier et UCLAF au sein de la CE). En parallèle, la décennie 1990 est marquée par le mouvement de judiciarisation des actions de dénonciation publique des corruptions et fraudes. Certains juges nationaux se plaignent, dans l'arène médiatique, des nombreux obstacles juridiques et matériels à la poursuite des délinquances économiques et financières ${ }^{24}$. L'Appel de Genève incarnera cette mobilisation de juges travaillant sur des affaires aux dimensions transnationales ${ }^{25}$. Ils sont relayés en cela par l'expertise des juristes européens qui, forts de cette médiatisation, promeuvent le Corpus juris et son incarnation, que serait l'instauration d'un Procureur européen ${ }^{26}$.

20 Passée cette phase de mise en place de l'OLAF au coeur d'intérêts stratégiques plus ou moins convergents, la décennie suivante est marquée par une rationalisation / routinisation des enquêtes, mais aussi par une intensification des critiques à son encontre.

21 Entre 2000 et 2004, le contexte est celui de l'élargissement de l'Union européenne à de nouveaux pays candidats connus pour leurs problèmes structurels de corruption et pour leurs débats en vue d'arrêter le texte de la future Constitution. Ces deux rendezvous politiques sont formulés de façon à poser les jalons d'une réforme de l'OLAF, auquel est associée stratégiquement l'idée du Procureur européen, présenté comme seul garant possible du contrôle de légalité du budget communautaire ${ }^{27}$. 
22 Le nouvel OLAF peine en effet, depuis son installation, à offrir des résultats aux observateurs extérieurs ${ }^{28}$. Pourtant, la mise en place d'un greffe et d'un système informatisé de gestion des dossiers d'enquêtes (internes et externes) appelé «Case Management System and Greff » (CMS) permet certes, un meilleur recensement des opérations et une amélioration notable du nombre de cas traités ${ }^{29}$, de même l'office fait des efforts considérables pour réduire la durée des enquêtes, depuis la phase d'évaluation jusqu'à celle d'investigation et de suivi. Cependant, il hérite de la gestion désordonnée des opérations engagées par son prédécesseur, l'UCLAF. C'est ainsi que le traitement d'environ 1400 cas a représenté un lourd héritage, de la même façon que l'affectation en bloc des personnels de l'UCLAF ne permet pas un changement radical de son management.

23 Aussi, l'année 2004 en tant que phase de discussion du traité de Convention européenne et contexte électoral propice aux batailles politiques, constitue à nouveau une période de forte remise en cause pour l'office. Certains voient le Traité comme l'opportunité de réformer l'OLAF et d'instaurer un cadre pour le futur Procureur européen ${ }^{30}$, d'autres proposent au contraire de fusionner Eurojust ${ }^{31}$ avec le futur Parquet européen ${ }^{32}$. Les conflits de vision entre la Commission, le Parlement et le Conseil sont à nouveau exacerbés. C'est dans ce contexte que l'office est directement mis en cause dans quatre « affaires » qui font douter de son impartialité et de son efficacité. Cette phase va ouvrir la voie à une seconde crise majeure qui contraint la Commission à proposer une nouvelle réforme de l'OLAF.

L'OLAF au coeur de polémiques à répétition

24 A la faveur de quatre polémiques illustrant une politisation évidente de l'office à travers les intérêts convergents ou divergents d'acteurs - selon qu'ils appartiennent au Comité de surveillance, à l'OLAF, à la Commission ou au Parlement européen -, les médias européens vont relayer des informations souvent ambiguës et/ou contradictoires, dont il est parfois difficile de deviner les émetteurs. Cependant ces " affaires" ont pour vertu de mettre en évidence les lacunes structurelles de l'OLAF, qui tiennent aux statuts ambigus lui servant de fondations précaires, compte tenu des circonstances de sa création dans le cadre de la crise Santer.

La première affaire est sans doute la plus lourde de conséquences puisqu'elle n'est pas encore close en 2006. L'affaire "Eurostat " héritée de son successeur, va, dès 2003, entacher la politique de " tolérance zéro » décrétée par Romano Prodi, le successeur du démissionnaire Jacques Santer. En mars 2004, le scénario de 1999 semble donc se rejouer : soixante-seize parlementaires appellent à une motion de censure mettant en cause la Commission, accusée de ne pas assumer ses responsabilités. Cependant, le nombre de signatures sera insuffisant pour que la motion soit présentée, les deux principaux partis s'étant abstenus.

26 L'OLAF et, à travers lui, la Commission dont il dépend fonctionnellement, est directement mis en cause dans cette affaire puisqu'on lui reproche de ne pas avoir communiqué aux institutions européennes les avancées de l'enquête concernant l'office de statistique européen, alors même que le cas était connu depuis mars 2000 dans le cadre des dénonciations faites par le fonctionnaire Van Buitenen ${ }^{33}$. Celles-ci avaient d'ailleurs initiées la première crise relayée par des acteurs intéressés par l'opportunité du scandale ${ }^{34}$.

27 Le principal volet de l'affaire "Eurostat » concerne les points de vente de données statistiques qui constituaient un dispositif de «réserves occultes» mis en place en 
Espagne, au Luxembourg et en Belgique. Des comptes secrets auraient été alimentés par le produit des ventes de données, constituant une enveloppe financière échappant au contrôle de la Commission. L'enquête révèle que ces réserves cachées furent utilisées pour payer «des frais de représentation et des frais d'experts pour des missions diverses ", ainsi que le loyer d'un sous-traitant d'Eurostat. Les fonds secrets ont permis aussi d'avantager l'un des principaux sous-traitant d'Eurostat, la société parisienne Planistat, "qui avait obtenu des marchés de service en soumettant des offres sousévaluées", sachant qu'elle obtiendrait des compensations. De même, le CESDCommunautaire, autre sous-traitant d'Eurostat au Luxembourg, dont l'un des dirigeants n'était autre que le directeur général d'Eurostat, a aussi été avantagé, avant qu'il n'abandonne la présidence fin 1994. L'existence de conflits d'intérêts, l'utilisation irrégulière des subventions octroyées et de financements multiples auraient abouti à une gestion erratique, dont le coût est évalué à 4,2 millions d'euros de préjudice pour les communautés ${ }^{35}$.

En septembre 2003, le président de la Commission, Romano Prodi doit répondre de cette affaire devant le Parlement, qui met en cause les institutions de contrôle défaillantes du point de vue de la communication et de la transparence : l'ancienne DG audit devenue le Service d'audit interne, la DG ADMIN et OLAF ${ }^{36}$.

Il utilise l'opportunité de cette arène parlementaire pour relancer le débat sur l'introduction du Procureur européen et «l'externalisation d'un OLAF qui pourrait évoluer vers une fonction d'auxiliaire de justice » dans le domaine administratif et financier.

30 Cette affaire, à travers la production abondante de rapports, auditions et recommandations auxquels elle a donné lieu, est utilisée pour renforcer la lutte contre les fraudes. Elle inaugure l'opportunité d'un nouveau tournant dans la perception et la construction du problème de l'évasion des fonds communautaires, cela à travers le rapport Bosch de mars 2004 qui oriente le débat sur la responsabilité des pays membres. En effet, il propose que les Etats membres fassent une déclaration annuelle d'assurance, certifiée par leur instance de contrôle national sur l'utilisation faite des dépenses communautaires. Le Parlement européen ne votera pas cette proposition mais celle-ci a le mérite de recentrer le débat sur la responsabilité des véritables débiteurs des politiques européennes que sont les Etats. Surtout les fonctions de contrôleurs économiques et financiers sont mises sur le devant de la scène, comme réaffirmées par le rapport spécial de la Cour des comptes en août 2005.

L'affaire Tillack, concomitante de celle d'Eurostat, met en évidence d'autres dysfonctionnements d'OLAF. Elle est déclenchée par une perquisition de la police belge dans les appartements de Hans-Martin Tillack, correspondant belge du magazine Stern, obligé de répondre aux accusations de "corruption d'un fonctionnaire d'OLAF » aux fins d'obtenir des informations qui auraient violées le secret de ses enquêtes. Vraisemblablement, les enquêteurs d'OLAF persuadés de fuites au sein de leurs effectifs, vont accuser ce journaliste d'avoir soudoyé l'un des leurs. La réponse àl'interprétation faite de cette accusation, relayée par les associations de journalistes, est que la liberté d'expression a été violée mais surtout que les droits de la personne à répondre de ses accusations n'ont pas été respectés. Cette affaire, non élucidée, ternit encore les affaires des nouveaux mandataires (nouveau Comité de surveillance et nouveau mandat pour son directeur général) de l'organisation en mars 2006, puisque l'OLAF a ouvert une enquête au sujet de l'origine de cinq documents internes de 
l'ancien Comité de surveillance ou de son secrétariat qui auraient été joints à la plainte portée contre OLAF par les membres d'Eurostat auprès du Tribunal de première instance ${ }^{37}$.

32 Le Comité de surveillance, dont le cheval de bataille, depuis sa constitution, concerne le respect des droits et libertés individuelles dans les enquêtes internes et qui déplore l'absence de procédure permettant un encadrement indiscutable des actes menés par OLAF, va se saisir de cette affaire pour réaffirmer ses principes. L'un de ses membres déclare qu'ils n'auraient pas été informés de la communication de ce cas aux autorités judiciaires ${ }^{38}$. Cette prise de position va cristalliser le conflit latent entre le Comité de supervision et la direction d'OLAF. En effet, cette affaire illustre une incohérence générique des règlements présidant au fonctionnement du CS, autorité consultative chargée de veiller au respect de l'indépendance d'OLAF et des droits des justiciables. Cependant, son règlement ne lui confère aucun pouvoir de décision. En ne se prononçant que sur les questions de principe, il établit une doctrine qui reste sans effets directs sur les enquêtes. Enfin, comme il ne peut intervenir dans le déroulement des enquêtes, il ne constitue en aucune manière un élément de contrôle des actes d'OLAF au cours de leur exécution.

Cette autorité n'a donc aucun pouvoir pour assurer un "contrôle de légalité » des actions menées par OLAF. Seuls le Tribunal de première instance et la Cour de justice européenne peuvent assurer ce contrôle mais a posteriori, une fois les violations portées en justice, et non pas de manière préventive au fil des enquêtes.

Une autre institution européenne, le médiateur européen, va aussi prendre parti dans cette polémique entre le journaliste et OLAF. Mobilisé par le plaignant, le médiateur reproche à l'OLAF dans son rapport de mai 2003 au Parlement européen, sa mauvaise administration de l'affaire lorsqu'ont été rendues publiques des accusations de corruption ne s'appuyant pas sur des bases factuelles. Il accuse plus explicitement l'OLAF de lui avoir communiqué des informations inexactes entre mars 2002 et mars 2003 , alors qu'il était saisi de cette affaire ${ }^{39}$. La direction d'OLAF maintiendra ses positions, mais cette affaire aura pour conséquence de modifier la législation belge sur la protection des sources.

En résumé, cette affaire illustre les mauvaises relations développées entre la direction d'OLAF et le Comité de Surveillance qui a toujours orienté le débat vers les questions de protection des droits des personnes ainsi que vers l'institutionnalisation du Corpus juris surtout portée par deux de ses membres : Mireille Delmas Marty et Brutus Liberati depuis 1997. Elle met aussi en évidence la faiblesse des organes consultatifs que sont le médiateur européen et le Comité de surveillance dont les recommandations sont systématiquement ignorées par la direction d'OLAF.

Une troisième affaire concernant des accusations de financement du terrorisme grâce aux fonds européens destinés à l'autorité palestinienne vont définitivement connoter politiquement les enquêtes de l'OLAF. En janvier 2004, ses enquêteurs sont envoyés à Jérusalem pour faire toute la lumière sur la gestion de ces fonds, suite aux allégations du FMI (Fonds monétaire international) concernant 900 millions d'euros qui auraient servi au financement illégal du terrorisme. Une commission d'enquête parlementaire constituée la veille de l'ouverture de l'enquête par l'OLAF, attend le retour des enquêteurs qui n'ont pas trouvé de preuves. Hypothèse peu probable puisqu'ils n'ont pas de pouvoir de perquisition. Leur rapport met en cause le manque de transparence 
dans la gestion de l'autorité palestinienne, mais, faute de preuve de détournement au profit des terroristes, un rapport minoritaire est conclu à une voix près.

Cette affaire illustre cette fois-ci le déficit de moyens opérationnels des enquêteurs d'OLAF. L'Office ne peut conduire que des enquêtes administratives. Ses enquêteurs peuvent faire des copies de documents dans les locaux des opérateurs économiques si tant est qu'on veuille bien leur ouvrir les portes, mais ils ne peuvent perquisitionner les domiciles de leurs propriétaires ou de leur personnel. Ils n'ont pas non plus accès aux comptes bancaires d'un opérateur économique ou d'un particulier, fût-il agent d'une institution communautaire. Ces limites tiennent à l'absence de personnalité juridique de l'office et affectent substantiellement la portée des enquêtes.

En revanche, un jugement de la Cour de justice de mars 2003 réaffirme le droit pour l'OLAF de conduire des recherches dans toutes les entités concernées, même des entreprises privées, qui auraient bénéficié de contrats européens. Elle déboute en ce sens la Banque centrale européenne et la Banque européenne d'investissement qui avaient tenté d'échapper aux investigations potentielles d'OLAF ${ }^{40}$. De même, les enquêteurs peuvent fouiller les bureaux des parlementaires européens sans mandat d'arrêt. Dans ce cas, ils peuvent porter plainte devant leurs tribunaux nationaux mais pas devant la CJE ${ }^{41}$.

39 Outre ces difficultés juridiques, cette affaire met aussi à jour la confusion qui règne autour des institutions européennes reconnues comme légitimes pour enquêter sur des suspicions de détournement de fonds. En effet, deux initiatives d'enquêtes parlementaires sont concomitantes de la prise en charge par l'OLAF : les présidents des commissions parlementaires des Affaires étrangères et de la Cocobu proposent la formation d'un groupe de travail, de même qu'une commission d'enquête parlementaire est déposée par des parlementaires européens ayant recueilli le quorum de voix requis. Cette situation de compétition entre les organes de contre-pouvoirs parlementaires et l'agence supposée indépendante qu'est l'OLAF illustre le déficit de confiance qui existe à son encontre en février 2003.

Enfin, une dernière affaire, aux retentissements plus modestes, va marquer un printemps 2004 particulièrement mouvementé pour OLAF. L'affaire "Dragon bleu » concerne une plainte de deux Français, directeurs d'une compagnie hydraulique, qui dénoncent des irrégularités dans la manière dont l'Union européenne a alloué des fonds, payés alors même que le projet n'a jamais véritablement été mis en place. En 2002, alors que le dossier était clos par OLAF, ils avaient déjà prononcé une plainte auprès du médiateur européen, accusant l'OLAF d'incapacité et, surtout, de mauvaise foi dans une enquête laissée au point mort. Le médiateur Nikiforos Diamandourios recommande de rouvrir ce dossier ou d'ouvrir une nouvelle enquête sur la base du témoignage des plaignants ${ }^{42}$. Cependant, cette recommandation restera lettre morte pour la direction d'OLAF, qui refuse de la suivre ${ }^{43}$.

41 Cette dernière affaire, cumulée aux précédentes, reflète l'absence récurrente de contrepouvoir face à l'OLAF. Celui-ci est insuffisamment contrôlé alors même que des dérives sont dénoncées dans les médias, par le Comité de surveillance et par le médiateur européen. La présomption d'innocence ou les droits de la défense ne semblent pas toujours respectés et les avis consultatifs de ces deux organes sont systématiquement ignorés.

Par ailleurs, l'influente et batailleuse Commission de contrôle budgétaire (Cocobu), qui représente un contre-pouvoir parlementaire, est accusée alternativement de politiser 
les affaires de mauvaises administrations de fonds européens en portant des dénonciations visant à déstabiliser la Commission et/ou certains commissaires ${ }^{44}$, alors que, dans le même temps, elle est délégitimée par des suspicions de "collusions culturo-institutionnelles " qui annihileraient ses fonctions. En effet, le commissaire responsable de la lutte contre les fraudes, Monsieur Schreyer est allemand, la présidente de la Cocobu, Madame Theato de même, tout comme les directeurs d'Europol et d'OLAF.

C'est donc dans ce contexte rendu confus par les batailles politiques se jouant dans le cadre des oppositions entre Parlement européen, Commission et Conseil sur le projet de Constitution européenne, et dans celui de la campagne électorale de juin 2004, qu'est envisagée la réforme de l'OLAF.

La Commission, afin de gérer la crise, propose une régulation amendée d'OLAF présentée en février 2004, sur laquelle s'était déjà engagé le président Prodi face à la Cocobu le 18 novembre 2003, lors de la présentation du programme législatif de la commission pour 2004. Pourtant, nous sommes forcés de constater que la proposition du président, qui laissait entrevoir une réorganisation de l'office lui-même allait bien plus loin que les aménagements finalement apportés aux procédures d'enquêtes ${ }^{45}$. Le contenu de la réforme présentée par la commission Prodi est donc discutable, mais surtout très timide par rapport à ce qui avait été au annoncé départ.

Cependant, l'arrivée de la nouvelle commission menée par le portugais Barroso et la nécessité de simplifier l'environnement fiscal pour répondre aux critères d'une compétitivité accrue afin d'atteindre les objectifs de la stratégie de Lisbonne à partir de novembre 2004 vont opérer un revirement radical vers l'intergouvernementalisme, jugé moins contraignants pour des Etats en mal de souveraineté. Le renforcement de l'OLAF est abandonné ${ }^{46}$.

Les nouvelles orientations du débat : la responsabilité des Etats membres dans la lutte contre l'évasion des fonds communautaires

Le paradigme de la protection des intérêts économiques et financiers des Communautés européennes, institutionnalisé grâce à la Convention de 1995, a servi de cheval de Troie à l'imposition de l'idée selon laquelle un procureur européen consacrerait l'intégration des espaces judiciaires européens, cela depuis 1997 et la création du Corpus juris vision qui fait cause commune avec les revendications de certains magistrats nationaux, mais aussi des représentants de la Cocobu du Parlement européen et ceux de la Commission. Néanmoins, plusieurs prises de positions institutionnelles tendent à confirmer l'imposition de l'hypothèse intergouvernementale depuis le début de la nouvelle décennie.

Le refus des chefs d'Etat et de gouvernement, en décembre 2000, d'intégrer un article dans le traité de Nice destiné à créer une base juridique pour adopter le Corpus juris marque le début de la réaffirmation des prérogatives étatiques en matière de lutte contre les phénomènes de criminalité. Ils optent pour un instrument de coopération, Eurojust. Désormais, la rhétorique de la coopération judiciaire, policière et de la collaboration des organes de contrôle nationaux s'impose dans les recommandations pour réformer l'OLAF.

48 En mars 2004 le rapport Bosch sur la protection des intérêts financiers de l'UE ${ }^{47}$ réoriente le débat sur la réforme d'OLAF vers la structure objective des problèmes à régler, à savoir que ce sont en fait les Etats membres qui détiennent les outils opérationnels d'une gestion rigoureuse des fonds communautaires, d'un contrôle 
effectif de ces mêmes dépenses effectuées sur leur territoire et surtout, des moyens de contraintes, pénaux et juridiques, à même de prévenir et punir le développement de ces fraudes. Cette perception du problème préside au tournant intergouvernemental donné à la protection des intérêts économiques et financiers des communautés. Elle avait été largement occultée dans la phase de mise en place d'OLAF.

En juin 2004, le projet de traité établissant une Constitution pour l'Europe réaffirme la règle de l'unanimité ${ }^{48}$. La création d'un ministère public européen reste donc soumise au régime intergouvernemental et à la règle de l'unanimité dans le projet du Praesiudium.

Pire, le traité de Prüm, signé le 27 mai 2005 par sept Etats, attaque de front les projets européens d'intégration dans les domaines de justice et de sécurité en instituant un échange privilégié de données en matière de coopération afin de lutter plus efficacement contre la criminalité transfrontalière, le terrorisme et l'immigration illégale. Ce traité, signé en dehors de l'espace institutionnel européen, est interprété comme une atteinte directe aux principes énoncés en octobre 2005 dans le programme quinquennal de La Haye qui pose les jalons de la coopération judiciaire jusqu'en $2010{ }^{49}$. Il illustre une nouvelle préférence pour l'arène intergouvernementale, voire extracommunautaire, concernant le champ opérationnel des questions de justice, répression et sécurité. Il peut aussi être vu comme une alternative trouvée à l'échec de communautarisation du 3e pilier.

51 De plus, le rapport spécial de la Cour des comptes d'août 2005 confirme l'abandon d'une modification statutaire d'OLAF pour lui privilégier quelques aménagements réglementaires ainsi qu'une externalisation de certains de ses services qui devraient retourner à la Commission ${ }^{50}$. Nous sommes loin des déclarations réformatrices de l'année 2004. La Cour des comptes insiste sur les effets des enquêtes d'OLAF jugées comme plus procédurales qu'orientées vers des exigences de qualité, une bonne gestion des ressources matérielles, humaines et financières. La CC met en évidence l'insuffisance des recouvrements effectifs des sommes détournées, mais également l'absence de sanctions disciplinaires et pénales infligées aux auteurs de fraudes. En cela, elle insiste sur la coopération nécessaire des services des Etats membres qui, dans l'état actuel des choses, sont lents voire absents lorsqu'il s'agit de fournir des données de qualité suffisante pour être exploitées. Elle évoque à ce propos les lacunes des opérations de coordination et d'assistance, mal assumées par l'office. Enfin, concernant le suivi judiciaire insuffisant des enquêtes ${ }^{51}$, elle propose de renforcer le rôle des magistrats rattachés depuis 2003 à l'office, cela dès l'ouverture des enquêtes afin de renforcer les éléments de preuves nécessaires pour transmission des dossiers aux autorités disciplinaires et judiciaires. Remarquons cependant que l'Unité de magistrats au sein d'OLAF reste très discrète et fait peu l'objet des débats sur la réforme.

Le rapport de la CC soulève donc des questions de fond, celle de la valeur ajoutée de l'OLAF au regard de la médiocrité des résultats obtenus mais, surtout, celle du déficit de coopération pour lui assurer quelque succès. La responsabilité des Etats dans la lutte contre l'évasion des fonds européens est, pour une fois, clairement posée. Seul un contexte de désinvestissement dans le projet de Corpus Juris attaché à celui d'OLAF depuis sa création a permis d'aborder plus objectivement et de manière empirique les données du débat. Ce rapport confirme le virage intergouvernemental en matière de lutte contre les délinquances financières au niveau européen, en ce qu'il justifie l'annulation des ambitions réformatrices de la commission Prodi par la commission 
Barroso qui lui succède et, dans le même temps, sanctionne les piètres résultats d'OLAF en renvoyant en partie aux Etats la responsabilité de ceux-ci.

De la même manière, la composition du nouveau Comité de surveillance nommé en novembre 2005 par le PE, la CE et le Conseil laisse à penser que l'accent mis sur les questions judiciaires et pénales n'est plus à l'ordre du jour. Le mandat du nouveau Comité de surveillance, qui a pris ses fonctions en février 2006, risque d'être difficile, étant donné ses bases règlementaires très fragiles. Sa nouvelle présidente, issue du Serious Fraud office, apportera de toute évidence un souffle nouveau, fort différent du précédent CS, critiqué pour son ingérence dans l'affaire Tillack, mais aussi pour son manque de soutien au Directeur général d'OLAF et pour avoir privilégié la destination pénale des enquêtes internes au détriment des externes. Dès son entrée en poste, la nouvelle présidente s'est appliquée à sécuriser les données du secrétariat du Comité, suspecté d'avoir été l'objet de fuites. Prenant en compte les relations conflictuelles de ses prédécesseurs avec la direction de l'office, elle semble déterminée à rétablir de bonnes relations intra-institutionnelles ${ }^{52}$. On peut supposer qu'une vision peut-être davantage opérationnelle et policière primera, quand bien même la coexistence avec l'influente Madame Theato, l'ancienne présidente de la Cocobu, reste imprévisible. La réforme de l'OLAF n'est évoquée que tardivement, fin mai 2006, depuis que la nouvelle commission a pris ses fonctions. La proposition vise essentiellement à modifier la gouvernance de l'office afin de lui assurer davantage de soutien politique, soutien qui lui a fait défaut jusqu'ici. On peut cependant craindre que le résultat soit à l'opposé de celui escompté puisque son indépendance sera d'autant plus mise en doute par les obsvervateurs extérieurs aux institutions européennes. En revanche, le nouveau commissaire responsable de la DG administration, audit et Lutte anti-fraude, Siim Kallas, a lancé quatre initiatives dans le domaine de la transparence concernant les commissaires européens (adoption d'un code de conduite et déclaration financière de leurs intérêts financiers), la publication d'un registre des groupes lobbyistes travaillant auprès des institutions européenne (conséquence de l'affaire Eurostat entre autres), la production de plus d'information sur les bénéficiaires des fonds communautaires, une orientation vers la stratégie classique et discutable du "shaming and punishing », et la publication de listes noires d'entités entachées par des affaires de fraudes.

Par ailleurs, début février 2006, la confirmation du renouvellement du mandat du directeur de l'OLAF, à la suite d'un processus de candidature litigieux ${ }^{53}$, confirme le soutien de la nouvelle commission Barroso et du Conseil ${ }^{54}$, à Franz-Hermann Brüner. Cette décision accompagne aussi la réaffirmation des priorités opérationnelles plutôt que légalistes qui l'ont opposées au précédent Comité de surveillance.

Ainsi, l'échec du traité de Convention européenne, ajouté à l'adoption de la Convention de Prüm, et les prises de position d'une Commission aux accents néo-libéraux associées au nouveau Comité de surveillance et à la reconduction de l'ancien directeur d'OLAF pourtant décrié, s'inscrivent dans un contexte de crainte de voir les prérogatives étatiques liées à l'exercice légitime du pouvoir de coercition entamées par la création d'un espace judiciaire européen davantage intégré. De toute évidence, à partir du printemps 2006, la relecture des problèmes de fraudes qui s'amorce à travers la dénonciation des problèmes d'évasion fiscale et d'insuffisance de la coopération administrative avec les pays membres, inaugure un tournant majeur dans la perception du problème et des options politico-économiques qui vont y être attachées. Cette préférence pour la thèse intergouvernementale de la construction européenne et la 
focalisation sur ses aspects financiers et fiscaux est le fruit du nouveau décryptage qui prévaut en matière de construction européenne, à savoir un détournement des questions fondamentales de politiques judiciaires pour lutter contre les fraudes et, surtout, un réinvestissement sur les aspects économiques et libéraux du marché unique où le plus petit dénominateur commun prévaut.

Fruit d'une histoire européenne controversée et d'un contexte d'urgence pour rétablir la légitimité de la Commission suite à la dénonciation des pratiques de mauvaises gestion de la commission Santer en 1999, la politique de lutte contre les fraudes entachant la gestion des fonds européens demeure cinq ans plus tard, associée à celle des scandales qui ébranlent régulièrement les institutions européennes. Les controverses et polémiques semblent présider aux premiers pas difficiles de l'office, qui a servi initialement de cheval de Troie aux ambitions de communautarisation du $3 \mathrm{e}$ pilier. Cette récurrence des scénarios laisse à penser que l'instrumentalisation des enquêtes pour fraudes dans le secteur médiatique pouvant délégitimer les institutions européennes et ses acteurs ont été un outil de mise sur agenda de réformes visant à soutenir des visions divergentes de la construction européenne. Une telle politisation de la protection des intérêts financiers européens est toutefois fort dommageable pour l'OLAF et les institutions européennes. Elle n'a, de ce point de vue, certainement pas répondu aux attentes initiales qui étaient de redresser le capital de confiance dans les institutions européennes ${ }^{55}$.

L'OLAF ne cumule pas les garanties d'indépendance nécessaires à la mise en application de la protection des intérêts économiques et financiers européens, que ce soit du point de vue de son indépendance fonctionnelle (budget et statut de ses personnels et directeur), de son imputabilité (absence de contrepouvoirs effectifs), des garanties juridiques qu'elle n'offre pas et de ses effets punitifs renvoyés à la responsabilité des Etats. En somme, cet office anti-corruption ne possède pas les moyens d'exercer un contrôle effectif des ressources communautaires.

La réforme avortée d'OLAF à ce stade consacre par ailleurs une tendance à la segmentation et à la parcellisation des initiatives visant à créer une politique européenne de la sécurité peu cohérente, reposant sur une coopération insuffisante et donc vouée à des difficultés inextricables de mise en oeuvre des mesures décidées.

Le développement d'une stratégie anticorruption est donc lié à la réalisation d'un projet non consensuel concernant la construction d'un espace judiciaire européen. La lutte antifraude est avant tout, au regard des développements les plus récents, instrumentalisée comme stratégie pour assurer une certaine idée de la gouvernance européenne plus ou moins intégrative ou minimaliste selon les commissions dans un contexte de mise sur agenda de la problématique sécuritaire. Elle relève de la politique $\mathrm{du}$ symbolique en incarnant alternativement une volonté de renforcement institutionnel ou au contraire un instrument d'intégration pour le marché européen, alors que les moyens effectifs qui lui sont donnés demeurent dépendants des gouvernements peu enclins à légiférer sur les questions de délinquances économiques et financières. On saurait donc prédire, étant donné sa genèse, que l'introduction de ce nouveau registre de légitimation (la lutte contre l'évasion des fonds communautaires) établi sur un désajustement entre la fin (le renforcement de la CE et du PE ou au contraire leur affaiblissement plus récent) et les moyens (les décisions intergouvernementales du 3e pilier), contribuera à entamer encore davantage le déficit de légitimité des institutions européennes. 


\section{NOTES}

1.. Entretien avec un enquêteur de l'OLAF, décembre 2000.

2.. Nous utiliserons dans cet article le sigle OLAF qui est l'acronyme officiel de l'office de lutte anti-fraude.

3.. Pujas V., « The European Anti-Fraud Office : a European policy to fight against economic and financial fraud?", Journal of European Public Policy, vol.10, n 5 , octobre 2003, pp. 778-797.

4.. Strengthening OLAF, the European Anti-Fraud Office, 24th Report of Session 2003-4, European Union Committee, House of Lords, 21st July 2004. Memorandum by Dr. Véronique Pujas, pp. 78-79.

5.. Nugent N., The Government and Politics of the European Union, Londres, Macmillan Press, 1999, p. 390.

6.. Janvier 1984, Doc. 1-1346/83

7.. Rapport Bosch : «Le Parlement inclut cinquante nouveaux postes dans le budget 1994 pour des agents de l'UCLAF et fait pression pour qu'une fonction précise de l'enquête leur soit attribuée. Il est obligé de reporter la décharge pour le budget 1992 pour forcer la Commission à prendre des mesures dans ce sens », p. 10 .

8. Commission d'enquête parlementaire sur le régime de transit.

9.. A4-0097/1998 - résolution informant la Commission des raisons du report de la décision de décharge sur l'exécution du budget général de l'Union européenne pour l'exercice 1997, adoptée le 31 mars 1998.

10.. OJC C348 of 18th November 1997, Court of Auditors covering 1996, vol.2.

11.. COM (87) 572 et COM (87) PV891.

12.. « Fraud and the EU budget », DG Research, PE 167.114, p. 9.

13.. " The European Council underlined the importance of continuing to combat fraud and irregularities in connection with the Community budget, both in view of the sums involved and in order to promote confidence in the construction of Europe... ", Copenhagen Council stated in June 1993.

14.. OJC C 316 OF 27.11.95, EP résolution du 19.9.96, OJ C320, 28.10.96

15.. Règl. $n^{\circ} 2988 / 95$

16.. Le premier protocole du 27 septembre 1996 définit la corruption portant atteinte aux intérêts financiers des CE et l'obligation des Etats membres de l'incriminer et de la sanctionner. Le protocole du 29 novembre 1996 concerne l'interprétation de la convention PIF et des ses protocoles par la Cour de justice.

17.. Le deuxième protocole du 19 juin 1997 porte sur l'obligation des Etats membres d'incriminer le blanchiment de capitaux et de prévoir la responsabilité des personnes morales, mais aussi la saisie et la confiscation des instruments et des revenus de ces infractions. Elle porte enfin sur les règles relatives à la coopération entre la Commission et les Etats membres et à la protection des données.

18.. Pujas V., « Les dispositifs nationaux de lutte contre les délinquances économiques et financières. Le cas italien », Revue de l'Institut des Hautes Etudes sur la sécurité intérieure, Paris, 2001, à paraître. Pujas V., « Les pouvoirs judiciaires en France, en Espagne et en Italie dans la lutte contre la corruption politique », Droit et Société, 44/45, juillet 2000. Pujas V., « Finanziamento dei partiti e controllo dei mezzi di communicazione : la specicità del caso italiano », in Pasquino G., Gilbert M. (ed.), 
Politica in Italia, Bologna, Il Mulino, 2000. Pujas V., Rhodes M., « Party Finance and Political Scandal in Italy, Spain and France », West European Politics, vol.22, n³, July 1999, pp. 41-63.

19.. Voir la Convention sur la protection des intérêts financiers des Communautés européennes dont le deuxième protocole n'a pas été ratifié par l'Italie, le Luxembourg et l'Autriche en juin 2006.

20.. www. europarl.eu, Working Paper, p. 20.

21.. Pujas V., 2003, ibid.

22.. Principal instrument de contrôle de l'exécution du budget reposant sur la rapport annuel de la Cour des comptes et sur la déclaration d'assurance.

23. Pour une étude détaillée de cet épisode voir Georgakakis D., 2000, « La démission de la Commission européenne : scandale et tournant institutionnel ? Cultures \& Conflits, n³8-39, pp. 39-59.

24.. Pujas V., « Les pouvoirs judiciaires en France, en Espagne et en Italie dans la lutte contre la corruption politique ", Droit et Société, 44/45, juillet 2000, pp. 41-60. Pujas V., « Délinquances économiques et financières et criminalité en cols blancs : les mutations contemporaines des conceptions de la déviance » in Roche S., Sociologie de la sécurité, Paris, Armand Colin, 2003, pp. 61-77.

25.. Robert D., La Justice ou le Chaos, Paris, Stock., 1996.

26.. Michel H., Mangenot M., Paris N., « Une Europe de la Justice », Arrêt sur recherches, $\mathrm{n}^{\circ} 5$, Mission de recherche Droit et Justice, juin 2005.

27. Livre vert sur la création d'un Procureur européen pour assurer la protection pénale des intérêts financiers communautaires, 11/12/2001, COM (2001)7515 final.

28.. Eu failing to stamp fraud, 27/04/01, BBC News.

29.. Evolution du nombre de dossiers enregistrés in Report of the European Anti Fraud Office, Supplementary Report for the year 2004. http://europa.eu.int/comm/ anti_fraud/reports/index_en.html

30.. Opinion 2/2002 on The Green Paper on the Criminal-Law Protection of The Community's Financial Interests and The Establishment of A European Public Prosecutor, 18/06/2002.

31.. Cet organe, créé le 28 février 2002, est voué à assurer la coopération entre les systèmes judiciaires et les Cours et tribunaux nationaux. Il doit être constitué de magistrats, de procureurs, de juges et d'autres experts judiciaires détachés de chacun des Etats de l'UE.

32.. Contribution présentée par M. Hubert Haenel, membre de la Convention : «Pour une véritable opérationnalité européenne dans la lutte contre la criminalité transnationale ». CONV 430/02

33.. Van Buitenen P., Fraudes à la Commission européenne, Castells-Labor, Paris, 2001.

34.. Voir l'analyse de Georgakakis D., 2000, op. cit.

35.. Le Monde, 25/09/03.

36.. SPEECH/03/432.

37.. Communiqué de presse OLAF, 9/03/2006

38.. Euobserver 20/04/04.

39.. Special Report from the European Ombudsman to the European Parliament following the darft recommandation to the European Anti-Fraud Office in complaint 2485/2004/GG.

40.. La BCE et la BIE soutenaient en 2000 qu'elles n'étaient pas soumises à l'obligation de permettre les enquêtes de l'OLAF au sein de leurs administrations respectives parce 
que le règlement 1073/99 ne leur était pas applicable. En effet, ne gérant pas de crédits communautaires, leurs représentants prétendaient qu'ils n'étaient pas concernés par le règlement. La Cour de justice européenne saisie par la Commission européenne a débouté les deux banques.

41.. Opinion of Advocate General Jaconbs delivered on November, the 20th 2003-Case C-167/02 P et Judgement by the Court of Justice on the investigative power of OLAF in the European Parliament, 30/03/2004, IP/04/424.

42.. Draft recommandation to the European Anti-Fraud Office in complaint 1769/2002/ (IJH)ELB

43.. Euobserver 23/03/2004.

44.. Concernant cette thèse voir l'article de D. Georgakakis, « La démission de la Commission européenne. Partie $2 »$, Culture \& Conflits, $n^{\circ} 38-39,2000$, pp. 59-71.

45.. Avis $n^{\circ} 6 / 2005$ de la Cour des comptes sur la proposition du Parlement européen et $\mathrm{du}$ Conseil modifiant les règlements relatifs aux enquêtes effectués par l'OLAF.

46.. A l'heure de clore cet article, le commissaire Siim Kallas vient de proposer quelques réformes mineures pour l'OLAF : la mise en place d'un conseiller réviseur indépendant qui se saisirait des plaintes dans le cours d'une enquête, un nouveau régime (non précisé) pour les enquêtes longues, l'information des institutions et personnes sur lesquelles OLAF enquête, la notification par les Etats membres des suites données aux enquêtes d'OLAF mais, surtout, la constitution d'une nouvelle gouvernance pour l'Office, à savoir, des réunions régulières entre le Comité de surveillance, des représentants du Parlement, du Conseil et de la Commission « pour discuter des priorités stratégiques d'enquête, garantir les bonnes relations entre les institutions et assurer l'efficacité du travail de l'OLAF » Communiqué de presse Europa, 24/05/2006. Cette proposition valide la thèse de l'abandon d'une réforme de fond et la politisation de l'office développée ci-dessous.

47.. Rapport Bosch, op. cit., p. 11.

48.. Dans les titre 6 chap. IV portant respectivement sur l'Espace de Liberté, de Sécurité et de Justice et, titre 4, chapitre II, la Lutte contre les Fraudes.

49.. Balzaq T., Bigo D., Carrera S., Guild E., Security and the Two-Level Game : The Treaty of Prüm, the EU and the Management of Threats, CEPS Working Paper $n^{\circ} 234$, janvier 2006.

50.. Cour des comptes, 2005, op. cit.

51.. « Le nombre de transmissions aux autorités judiciaires varie fortement d'une année à l'autre (10 en $2002 ; 43$ en $2003 ; 7$ pour le premier semestre 2004). Sur 57 décisions négatives relevées, 33 étaient motivées par l'insuffisance de preuves » in Rapport d'activités du Comité de surveillance, 22 décembre 2004, p. 30.

52.. Voir le discours de sa nouvelle présidente Rosalind Wright, 22/02/06. Site web d'OLAF : http://ec.europa.eu/comm/anti_fraud/index_fr.html.

53.. La campagne de recrutement pour son poste a été ouverte de manière interne pendant le seul mois de décembre 2005. Devant le mécontentement du Parlement et des médias, le commissaire Siim Kallas décide alors sous la pression de rouvrir la candidature en janvier 2006. Le Suédois, Bjorn Eriksson reçoit alors les préférences du Parlement. Pourtant, le Conseil et la Commission soutiendront le directeur d'OLAF dont les positions sont connues malgré les critiques qui ont fusé contre lui, dans la gestion de l'affaire Tillack notamment.

54.. Les préférences du PE pour le candidat suédois sont ignorées. 
55.. Si l'on considère les trois derniers eurobaromètres (de l'été 2004, du printemps 2005 et de l'automne 2005) la dégradation du climat de confiance est symptomatique pour la Commission européenne. Elle passe successivement et respectivement de 27 à 31 et $33 \%$ de non confiants, alors que le nombre de confiants reste plus ou moins le même, tandis que, pour le Parlement européen, le pourcentage des défiants passe de 26 à 31 puis $34 \%$ alors que la confiance se dégrade de manière évidente.

\section{RÉSUMÉS}

Le processus d'émergence du paradigme de contrôle des fonds communautaires qui a trouvé une réalisation dans la création de l'office européen de lutte contre les fraudes (OLAF) en 1999, illustre les tentatives d'institutionnalisation d'un espace judiciaire européen. Depuis les débuts de la mise sur agenda des problèmes d'évasion des ressources propres, la formulation de la problématique est surtout le fait des organes financiers européens. Mais le contexte des années 1990, qui voit la dénonciation des délinquances économiques et financières portée par les juges, va infléchir le débat vers l'instauration d'un Parquet européen présenté comme seul garant possible d'un contrôle de légalité des fonds européens. Toutefois, les rendez-vous institutionnels successifs depuis le traité de Nice tendent à invalider cette vision et réorienter le débat vers la promotion de la coopération des agences de contrôle nationales. Le renforcement de l'OLAF, entaché depuis ses débuts par des controverses, apparaît bien compromis.

The emergence of a paradigm of control of European funds has brought about the creation of the European Anti-Fraud Office (OLAF) in 1999. This institutional innovation illustrates the attempt to institutionalize a European legal space. When the problem of evading the duties and levies which supply the community budget was first set on the agenda, it was usually raised by the European financial organizations. But the context of the 1990s sees judges getting more and more involved in the denunciation of economic and financial delinquency. Hence, the debate is marked by the proposal to create a European public prosecutor's department. The latter is in this context indeed seen as the sole organization able to control the legality of European funds. However, the institutional meetings since the Treaty of Nice have tended to invalidate this vision and to reorient the debate towards the promotion of cooperation between national control agencies. Thus the strengthening of OLAF, surrounded by controversies since its very creation, seems highly uncertain.

\section{INDEX}

Index géographique : Union européenne

Thèmes : Commission européenne

Mots-clés : bureaucraties/ théorie des organisations, construction européenne, institutions politiques et agences, protection 


\section{AUTEUR}

\section{VÉRONIQUE PUJAS}

Véronique Pujas est chercheuse CNRS détachée. Elle travaille sur la construction des problèmes de criminalité transnationale et leur traitement par les organisations internationales. Ses principales publications portent sur la dénonciation de la corruption, le rôle des juges, le financement illégal des partis politiques. 